\title{
ARTIGO ORIGINAL Prevalência de depressão entre homens adultos em situação de rua em Belo Horizonte
}

\author{
Prevalence of depression among adult men in the streets in Belo Horizonte \\ Nadja Cristiane Lappann Botti', Carolina Guimarães de Castro², Monica Ferreira da Silva², Ana Karla Silva², \\ Ludmila Cristina de Oliveira ${ }^{2}$, Ana Carolina Henriques Oliveira Amaral de Castro², Leonardo Leão Kahey Fonseca²
}

\section{RESUMO}

Objetivo: Este estudo objetiva avaliar a prevalência de depressão entre a população de rua. Métodos: Realizado estudo transversal com homens adultos em situação de rua de Belo Horizonte. Para coleta de dados, foi utilizado o Inventário de Depressão de Beck e análise foi

\section{Palavras-chave}

Depressão, saúde mental, população de rua, Inventário de Depressão de Beck.

\section{Keywords}

Depression, mental health, homeless people, Beck Depression Inventory. realizada por meio da estatística descritiva. Resultados: Evidenciam-se alta prevalência de depressão e o grau de severidade dos sintomas depressivos relacionados à faixa etária, tempo de moradia na rua, local onde dorme, relato de problema de saúde e uso de medicamento da população masculina adulta em situação de rua. Conclusão: $O$ presente estudo ressalta a importância de construção de políticas de saúde voltadas para a população de rua.

\section{INTRODUÇÃO}

Características comuns da população em situação de rua são o estabelecimento do espaço público da rua como campo de relações privadas e a vivência da exclusão social pelo trinômio: expulsão, desenraizamento e privação. A exclusão social inclui a situação extrema de ruptura de relações familiares e afetivas, além de ruptura total ou parcial com o mercado de trabalho, e de não participação social efetiva'. Nesse sentido, essa população é vítima dos processos sociais, po- líticos e econômicos excludentes². Define-se como grupo populacional heterogêneo que tem em comum a pobreza, vínculos familiares quebrados ou interrompidos, vivência de um processo de desfiliação social pela ausência de trabalho assalariado e das proteções derivadas ou dependentes dessa forma de trabalho, sem moradia convencional regular e tendo a rua como o espaço de moradia e sustento ${ }^{3}$.

Como fatores para a situação de rua, encontram-se os estruturais, biográficos e desastres de massa e/ou naturais. Trata-se de um fenômeno multifacetado que não pode ser

\footnotetext{
1 Universidade Federal de São João Del Rei (UFSJ).

2 Pontifícia Universidade Católica de Minas Gerais (PUC-MG), campus Betim, Departamento de Enfermagem. 
explicado por uma perspectiva unívoca e monocausal, pois são múltiplas as causas de se ir para a rua, assim como são múltiplas as realidades da população em situação de rua ${ }^{4}$.

As condições adversas de sobrevivência dos moradores de rua podem, também, desencadear problemas mentais orgânicos. Estudo realizado com homens e mulheres moradores de albergues públicos das cidades do Rio de Janeiro e Niterói (RJ) mostra a presença de distúrbios mentais maiores $(22,6 \%)$, esquizofrenia (10,7\%), depressão maior (12,9\%), déficit cognitivo grave (15\%) e abuso/dependência de álcool $(44,2 \%)$ decorrentes da adversidade a que estão submetidos ${ }^{5}$.

Os distúrbios mentais maiores aparecem com maior prevalência em homens solteiros em situação de rua. Em comparação a outros subgrupos, este tem um maior período de vivência nas ruas, aumentando o risco de agravamento de doença física e mental. Em geral, os distúrbios mentais maiores antecedem à condição de morar nas ruas, como também a condição precária de existência nas ruas pode exacerbar os seus sintomas anteriores, assim como favorecer o aparecimento de outros distúrbios, levando a uma alta prevalência de comorbidade nesses indivíduos, tornando os casos ainda mais graves ${ }^{6}$. Estudo realizado em Juiz de Fora (MG) com a população em situação de rua localizada em instituições e no próprio espaço público encontrou taxa de esquizofrenia acima da encontrada na população geral brasileira ${ }^{7}$.

Diante dessa realidade, em 2008 foi implantada no Brasil a Política Nacional para Inclusão Social da População em Situação de Rua, a fim de estabelecer diretrizes e rumos que possibilitem a (re)integração dessas pessoas às suas redes familiares e comunitárias, o acesso pleno aos direitos garantidos aos cidadãos brasileiros e o acesso a oportunidades de desenvolvimento social pleno. Entre as diversas ações propostas por essa Política, encontra-se no subitem saúde o "incentivo à produção de conhecimento sobre a temática saúde desta população e aos mecanismos de informação e comunicação"3-21. Partindo desse incentivo à produção de conhecimento sobre a temática saúde da população de rua e reconhecendo que ela se encontra vulnerável a vários fatores que podem desencadear doenças ${ }^{8}$, justifica-se a importância de investigar a prevalência de sofrimento mental nessa população. Segundo a Organização Mundial da Saúde (OMS), diversos fatores podem provocar transtornos mentais, entre eles citam-se: pobreza, sexo, idade, conflitos e desastres, a maioria das doenças físicas e o ambiente familiar e social. Desde a década de 1990, a depressão vem ocupando posição de destaque entre os problemas de saúde pública, considerada a principal causa de incapacidade em todo o mundo?.

Apesar de intensas tentativas para estabelecer as bases etiológicas e fisiopatológicas, a causa precisa dos transtornos depressivos ainda é desconhecida. Existe concordância de que múltiplos fatores etiológicos (genéticos, bioquímicos, psicodinâmicos e socioambientais) podem interagir de maneiras complexas e de que a compreensão do fenômeno depressivo requer entendimento das relações entre esses vários fatores. Em relação à depressão, encontram-se diversos estudos que analisam os efeitos dos fatores socioeconômicos ${ }^{10}$.

A Classificação Estatística Internacional de Doenças e Problemas Relacionados à Saúde, 10a Revisão (CID-10), classifica os episódios depressivos em: (a) leve: quando o indivíduo ainda é capaz de realizar grande parte de suas atividades diárias; (b) moderado: quando o indivíduo começa a apresentar dificuldades no prosseguimento de sua vida cotidiana; e (c) grave: quando há marcantes sentimentos de desvalia, baixa autoestima e ideias suicidas ${ }^{11}$.

A partir dessas considerações, este estudo objetiva avaliar a prevalência de depressão entre a população de rua adulta masculina em situação de rua da cidade de Belo Horizonte.

\section{MÉTODOS}

Refere-se a estudo de prevalência do tipo transversal realizado com a população adulta masculina em situação de rua de Belo Horizonte. Os participantes foram selecionados por meio de uma amostra aleatória, dentre a população em situação de rua que frequentou o Centro de Referência da População de Rua (CRPR) no período de março a julho de 2009.

A amostra foi composta por 245 homens adultos em situação de rua de Belo Horizonte. Essa amostra foi definida por ser o subgrupo mais prevalente na população que vive nessa situação em Belo Horizonte, de acordo com o estudo censitário realizado em 2005 (85,6\% homens contra 14,4\% mulheres) ${ }^{12}$, e também por serem os homens em situação de rua os que mais frequentam o CRPR, de acordo com o consolidado de frequência do ano de 2008 da Secretaria Municipal Adjunta de Assistência Social/Gerência de Abordagem Social/CRPR (86,1\% homens contra 13,9\% mulheres).

O estudo foi realizado no CRPR, serviço vinculado à Secretaria Municipal Adjunta de Assistência Social, localizado próximo à rodoviária na região central de Belo Horizonte e que tem por objetivo resgatar a autoestima e a identidade, além de incorporar valores e comportamentos para a superação do processo de ruptura e exclusão social do morador de rua. É um serviço diurno aberto à população de rua, com atividades diferenciadas de acordo com o turno. Pela manhã são realizadas oficinas socioeducativas, entre elas: futebol, teatro, cultura popular, ambientação, artes plásticas e cineclube. No período da tarde, o CRPR é aberto para lavação de roupas, banho e armazenamento de pertences pessoais. O acesso ao CRPR pela população em situação de rua se dá por encaminhamento feito pelos albergues e abrigos públicos da cidade e pela livre demanda da população em situação de rua.

Foram utilizados dois instrumentos para coleta de dados. Primeiro aplicou-se um questionário sociodemográfico para coleta de dados referentes a caracterização e condições de vida e de saúde inerentes da população em situação de rua. No segundo momento, aplicou-se o Inventário de Depressão 
de Beck (Beck Depression Inventory - BDI), que é um instrumento para a medida de sintomatologia de depressão muito utilizado na área clínica e em pesquisas, que possui vários estudos psicométricos com amostras brasileiras ${ }^{13}$. Trata-se de uma escala de autorrelato, para levantamento da intensidade dos sintomas depressivos, composta por 21 itens que abarcam os componentes cognitivos, afetivos, comportamentais e somáticos da depressão. O escore total permite a classificação dos níveis de intensidade da depressão. O ponto de corte utilizado foi 12 para diagnóstico de depressão ${ }^{14}$.

Para caracterização amostral, foram adotadas as variáveis: idade, estado civil e escolaridade. Foram classificadas como variáveis de estudo: faixa etária conforme a escala de classificação da OMS (idade adulta jovem - dos 15 aos 30 anos, idade madura - dos 31 aos 45 anos e idade de mudança - dos 46 aos 60 anos), faixa de tempo de moradia na rua, local onde dorme, relato de problema de saúde e uso de medicamento, grau de sintomas depressivos determinado pelo BDI. Os dados foram sistematizados em planilha do programa Microsoft Excel segundo frequência absoluta (n) e relativa (\%). Após o processamento dos dados, procedeu-se à análise estatística descritiva.

A coleta de dados foi realizada por quatro acadêmicos de enfermagem que participaram do estudo em sala reservada, localizada no CRPR, facilitando o contato com o entrevistado, garantindo privacidade às suas respostas. Para a coleta, foi elaborada uma escala que contemplasse dias da semana, de segunda a sexta-feira, no turno da manhã e tarde, durante o período de março a julho de 2009. A média de tempo para responder ao Inventário de Depressão de Beck foi de 30 a 40 minutos. Neste estudo foram considerados critérios de inclusão: idade de 18 anos ou mais, capacidade física e mental para responder ao questionário e aceitação concedida voluntariamente após explicação do objetivo deste estudo, sigilo do resultado e a segurança do anonimato.

O protocolo do estudo foi aprovado pelo Comitê de Ética em Pesquisa da Pontifícia Universidade Católica de Minas Gerais (PUC) (0212.0.213.000-07) e autorizado pelo CRPR e pela Secretaria Municipal Adjunta de Assistência Social. A participação dos sujeitos da pesquisa foi voluntária e livre de qualquer forma de remuneração. Os participantes consentiram em participar preenchendo o Termo de Consentimento Livre e Esclarecido.

\section{RESULTADOS}

O grau de escolaridade dos participantes variou entre analfabetos $(6,7 \%)$, com ensino fundamental incompleto $(39,5 \%)$ e completo $(26,7 \%)$, médio incompleto $(10,3 \%)$ e completo $(15,4 \%)$, superior incompleto $(1,0 \%)$ e completo $(0,5 \%)$. Quanto ao estado civil, foram identificados solteiros (66\%), casados/com companheiro (18,4\%), divorciados/separados $(14,1 \%)$ e viúvos (1,5\%). Em relação à faixa etária, verificou-se que $38 \%$ apresentam idade entre 15 e 30 anos, 42,9\%, entre
31 e 45 anos e 19,1\%, entre 46 e 60 anos. A idade média geral encontrada foi de 35,5 \pm 10,2 anos.

Quando analisados os pontos obtidos no BDI, verificou-se que 107 (43,7\%) moradores de rua obtiveram escores finais menores que 11 pontos, portanto foram classificados como sem sintomas depressivos, enquanto 138 (56,3\%) moradores de rua totalizaram acima de 12 pontos, sendo classificados como com sintomas depressivos. Entre os homens em situação de rua com presença desses sintomas, foi constatado grau leve em 66 (26,9\%), seguindo-se de grau moderado acometendo 60 (24,5\%) moradores e $12(4,9 \%)$ casos de sintomas depressivos graves. A ocorrência e a gravidade dos sintomas determinados por meio da aplicação do BDI podem ser visualizadas na tabela 1.

Tabela 1. Distribuição da frequência absoluta (n) e relativa (\%) de ausência ou presença de depressão em seus diferentes graus na população adulta masculina em situação de rua, de acordo com o BDI (Belo Horizonte, 2009)

\begin{tabular}{lll}
\hline Depressão & Frequência absoluta $(\mathbf{n})$ & Porcentagem (\%) \\
\hline Sem depressão & 107 & 43,7 \\
Com depressão leve & 66 & 26,9 \\
Com depressão moderada & 60 & 24,5 \\
Com depressão grave & 12 & 4,9 \\
Total & 245 & 100 \\
\hline
\end{tabular}

Podem-se notar, na tabela 2, os valores absolutos e percentuais dos diferentes escores (sem depressão, depressão leve, moderada e grave) entre as variáveis de estudo (faixa etária, faixa de tempo de moradia na rua, local onde dorme, relato de problema de saúde e uso de medicamento). Essa tabela aponta para o grau de severidade dos sintomas depressivos em relação à faixa etária, merecendo maior atenção os graus de depressão leve e moderada nos adultos maduros (21\% e $19,6 \%)$ e a depressão grave no adulto jovem (2,9\%) morador de rua. Observa-se maior associação da depressão leve, moderada e grave entre os homens que se encontram entre um e seis meses em situação de rua (10,9\%, 9,4\% e 2,9\%). Verifica-se maior prevalência dos sintomas depressivos leves, moderados e graves na população em situação de rua (31,2\%, 23,9\% e 5,1\%) que utiliza as instituições públicas de Belo Horizonte como local para dormir, quando comparada com a que dorme em vias públicas. Observa-se ainda prevalência de sintomas depressivos na população em situação de rua que declara problema de saúde $(52,9 \%)$ e uso de medicamento (29,7\%). Na população em situação de rua com depressão que declara problema de saúde, verifica-se maior frequência dos sintomas moderados $(23,9 \%)$ e leves $(23,2 \%)$. Por sua vez, dos que declaram uso de medicamentos, verifica-se maior frequência dos sintomas leves (13\%) e moderados (12,3\%). Verifica-se também o dobro da frequência de sintomas depressivos graves na população de rua que declara problema de saúde (5,8\%), quando comparada com a que declara não ter problema de saúde (2,9\%). 
Tabela 2. Distribuição da frequência absoluta (n) e relativa (\%) de ausência ou presença de depressão em seus diferentes graus, segundo a faixa etária, tempo de moradia na rua, local onde dorme, declaração de problema de saúde e uso de medicamento, entre a população adulta masculina em situação de rua, de acordo com o BDI (Belo Horizonte, 2009)

\begin{tabular}{|c|c|c|c|c|c|c|c|c|c|c|}
\hline & \multicolumn{2}{|c|}{ Sem depressão } & \multicolumn{2}{|c|}{ Com depressão } & \multicolumn{2}{|c|}{ Com depressão leve } & \multicolumn{2}{|c|}{ Com depressão moderada } & \multicolumn{2}{|c|}{ Com depressão grave } \\
\hline & $\mathrm{n}$ & $\%$ & $\mathrm{n}$ & $\%$ & $\mathrm{n}$ & $\%$ & $\mathrm{n}$ & $\%$ & $n$ & $\%$ \\
\hline \multicolumn{11}{|l|}{ Faixa etária } \\
\hline Adulto jovem & 36 & 33,6 & 57 & 41,3 & 26 & 18,8 & 24 & 17,4 & 7 & 5,1 \\
\hline Adulto maduro & 45 & 42,1 & 60 & 43,5 & 29 & 21,0 & 27 & 19,6 & 4 & 2,9 \\
\hline Adulto médio & 26 & 24,3 & 21 & 15,2 & 11 & 8,0 & 9 & 6,5 & 1 & 0,7 \\
\hline \multicolumn{11}{|l|}{ Tempo de moradia na rua } \\
\hline Até 1 mês & 11 & 10,3 & 9 & 6,5 & 4 & 2,9 & 4 & 2,9 & 1 & 0,7 \\
\hline Entre 1 e 6 meses & 19 & 17,8 & 32 & 23,2 & 15 & 10,9 & 13 & 9,4 & 4 & 2,9 \\
\hline Entre 6 e 12 meses & 16 & 15,0 & 15 & 10,9 & 8 & 5,8 & 6 & 4,3 & 1 & 0,7 \\
\hline Entre 12 e 36 meses & 13 & 12,1 & 21 & 15,2 & 8 & 5,8 & 12 & 8,7 & 1 & 0,7 \\
\hline Entre 36 e 72 meses & 20 & 18,7 & 26 & 18,8 & 13 & 9,4 & 12 & 8,7 & 1 & 0,7 \\
\hline Entre 72 e 120 meses & 11 & 10,3 & 17 & 12,3 & 9 & 6,5 & 5 & 3,6 & 3 & 2,2 \\
\hline Mais de 120 meses & 17 & 15,9 & 18 & 13,0 & 9 & 6,5 & 8 & 5,8 & 1 & 0,7 \\
\hline \multicolumn{11}{|l|}{ Local onde dorme } \\
\hline Instituição pública & 70 & 65,4 & 83 & 60,1 & 43 & 31,2 & 33 & 23,9 & 7 & 5,1 \\
\hline Via pública (rua) & 36 & 33,6 & 56 & 40,6 & 23 & 16,7 & 28 & 20,3 & 5 & 3,6 \\
\hline \multicolumn{11}{|l|}{ Problema de saúde } \\
\hline Não declara & 64 & 59,8 & 65 & 47,1 & 34 & 24,6 & 27 & 19,6 & 4 & 2,9 \\
\hline Declara & 43 & 40,2 & 73 & 52,9 & 32 & 23,2 & 33 & 23,9 & 8 & 5,8 \\
\hline \multicolumn{11}{|l|}{ Uso de medicamento } \\
\hline Não declara & 79 & 73,8 & 97 & 70,3 & 48 & 34,8 & 43 & 31,2 & 6 & 4,3 \\
\hline Declara & 28 & 26,2 & 41 & 29,7 & 18 & 13,0 & 17 & 12,3 & 6 & 4,3 \\
\hline
\end{tabular}

\section{DISCUSSÃO}

A maioria da amostra foi composta por homens solteiros (66,0\%), na faixa etária de 31 a 45 anos (42,9\%), com ensino fundamental incompleto (39,5\%), vivendo em situação de rua num período inferior a 12 meses $(41,7 \%)$. Características preocupantes, pois importante variável no que se refere a essa população diz respeito ao tempo em que se encontram nessa condição. Em grande medida, o tempo acaba por determinar as chances de um indivíduo sair da situação de rua; quanto menor o tempo de rua, normalmente, maiores as probabilidades, e, inversamente, quanto maior o tempo, menores as possibilidades ${ }^{15}$.

Entre os principais motivos pelos quais as pessoas passaram a viver e morar na rua encontram-se o alcoolismo e/ ou drogas, desemprego e desavenças familiares ${ }^{16}$. A situação de rua corresponde à condição extrema de miséria em que se têm negados os direitos fundamentais, representando a linha final do processo crônico de exclusão social. A população em situação de rua torna-se altamente vulnerável a múltiplos fatores de risco para a saúde, os quais estão associados à sua condição extrema de pobreza. Seus problemas físicos e mentais podem, em parte, ser explicados pela sua condição desfavorável de vida ${ }^{17}$.

Neste estudo, o rastreamento para depressão detectou prevalência de 56,3\%, taxa bastante elevada, revelando a fragilização da saúde dos homens moradores de rua de Belo Horizonte. A depressão pode ser definida como estado de alterações do humor envolvendo irritabilidade, tristeza profunda, apatia, disforia, anedonia, alterações cognitivas, motoras e somáticas. Os sintomas depressivos interferem no funcionamento social do indivíduo, bem como em outras áreas significativas da vida, como trabalho e relações pessoais ${ }^{18}$.

Pesquisas realizadas no Brasil revelam que a taxa de distúrbios mentais maiores nos moradores de albergues públicos é maior do que a taxa encontrada na população adulta em geral ${ }^{5}$. Destacam-se achados que colocam que os diagnósticos do grupo das psicoses esquizofrênicas constituem um subgrupo específico entre os moradores de rua, com características demográficas, biográficas e comportamentais próprias. Assim, estudos confirmam a alta prevalência de transtornos mentais entre os moradores de rua? ${ }^{7}$. 
A depressão é o quarto maior agente incapacitante das funções sociais e outras atividades da vida cotidiana, sendo responsável por cerca de 850 mil mortes a cada ano². Estudos colocam que a pobreza pode ser um fator de muita relevância na explicação do aumento dos transtornos do humor, pois eles estão associados a condições sociais como desemprego, baixo nível de instrução, falta de moradia e alimentação inadequada ou insuficiente. Tais condições, exclusivas ou combinadas, podem favorecer o desenvolvimento de um estado de desesperança, que tem como efeito imediato a redução da capacidade para lidar com situações estressoras de maneira adequada, reduzindo a disposição para suportar fatos adversos e frustrantes. Assim, a desesperança pode propiciar o estabelecimento de estados depressivos, ansiedade e redução drástica da autoestima ${ }^{19}$, características e condições inerentes à situação de rua, isto é, à população que sofre com a exclusão da sociedade, pois são indivíduos que em seu dia a dia suportam a desigualdade social. E sabe-se que quanto maior for a desigualdade social de um país, maior será a repercussão na qualidade de vida, consequentemente na saúde, da sociedade de forma geral, do sujeito e/ou de sua família em particular. Entre os estudos que avaliam o efeito das condições econômicas sobre a saúde mental da população em situação de rua propriamente dita, encontram-se pesquisas sobre a depressão em indivíduos desabrigados e sobre os efeitos do subemprego sobre a depressão ${ }^{20,21}$.

A prevalência do quadro de depressão encontrado neste estudo pode relacionar-se a outros comprometimentos psiquiátricos da população em situação de rua. Muitos são os distúrbios mentais que acometem os moradores de rua. Existe, entretanto, a dificuldade na delimitação do fator temporal de causalidade, ou seja, se os distúrbios antecedem ou sucedem o fato de morar nas ruas, fato que exige maiores investigações ${ }^{20,21}$. Sabe-se que há grande prevalência de alcoolismo na população de moradores de rua, em comparação à população em geral, tornando-a mais vulnerável, entre outros comprometimentos, aos problemas mentais relacionados ao abuso/dependência de álcool 20,21 .

Em relação à gravidade dos sintomas depressivos encontrados entre os homens em situação de rua, constata-se maior frequência dos graus leves e moderados. A prevalência de depressão encontrada neste estudo pode ser explicada pelo processo de exclusão social a que estão submetidos, que pode ser entendido como alto grau de vulnerabilidade psicossocial da população em situação de rua quando se reconhece o contexto adverso da situação de permanência na rua. Nesse contexto, verificam-se inúmeros fatores presentes na vida dos moradores de rua que comprometem a estrutura individual com agravo da capacidade mental e física, como a miséria, violência, consumo abusivo de drogas, rompimento com a família e situações de violência doméstica, sexual e moral22.

Para entender o processo de exclusão social da população em situação de rua, não se podem deixar de lado motivações socioculturais e históricas, isto é, historicamente a exclusão social abarca dois aspectos: as relações produtivas e as questões socioculturais e de cidadania ${ }^{23}$. Assim, as definições e os conceitos sobre exclusão social abordam tanto a esfera produtiva como as relações e vínculos sociais básicos. Excluir não significa apenas segregar, marginalizar, mas também desconsiderar a existência humana; significa "(...) criar e perpetuar condições que tornem permanente o ato de morrer"23-28. De acordo com essa concepção, devem-se considerar tanto o contexto social como o político, o cultural e o humano ${ }^{24}$.

A análise entre grau de severidade dos sintomas depressivos e faixa etária e tempo de moradia na rua revela maior frequência da depressão grave no adulto jovem morador de rua e da depressão leve, moderada e grave entre os homens que se encontram entre um e seis meses morando na rua. Pode-se entender essa gravidade pela associação de fatores psicodinâmicos e socioambientais. Nesse sentido, estudos colocam a depressão entre os transtornos que apresentam maior incidência em indivíduos que tiveram vínculos desfeitos durante a infância ${ }^{25}$. Na mesma vertente, há pesquisas que apontam mais frequência dos quadros depressivos após perdas significativas, entre elas a morte de pessoas, emprego, local de moradia, status socioeconômico, ou de algo puramente simbólico ${ }^{26}$.

Existem algumas classificações de moradores de rua; nesse grupo social há os que não possuem residência, os que moram em instituições públicas e os que vivem cronicamente nas ruas ${ }^{27}$. Também são várias as denominações encontradas para as pessoas que vivem nas ruas, tanto utilizadas pela própria população de rua como por pesquisadores ou agentes do poder público. Uma denominação muito utilizada pela própria população de rua é "sofredor de rua", que especifica o sofrimento que essa população vivencia nas ruas $^{28}$. Independente da denominação utilizada, é muito clara a imensa situação de vulnerabilidade a que a população de rua está exposta. Neste estudo verifica-se maior prevalência dos sintomas depressivos leves, moderados e graves na população em situação de rua que utiliza as instituições públicas de Belo Horizonte (Albergue Noturno Municipal Tia Branca, Abrigo São Paulo ou República Reviver) como local para dormir, quando comparada com a que dorme em vias públicas (em bancos, calçadas, viadutos, marquises, praças ou prédio invadido). Mais estudos são necessários para o entendimento desses achados, mas uma possibilidade da maior frequência de depressão entre os moradores de rua que utilizam as instituições da política pública da Assistência Social pode referir que nessa situação há a construção da identidade social de morador de rua.

Observa-se, ainda, prevalência de sintomas depressivos na população em situação de rua que declara problema de saúde e uso de medicamento. Estudos evidenciam entre os problemas de saúde dos moradores adultos, masculinos, em situação de rua de Belo Horizonte, os problemas psiquiátricos como principal enfermidade declarada, seguidos 
dos respiratórios, cronicodegenerativos, gastrointestinais/ hepáticos, neurológicos e infectocontagiosos ${ }^{12,28}$. Estudos chamam a atenção sobre a precariedade das condições de vida da população de rua. Nesse sentido, verifica-se que várias são as doenças que atingem com maior frequência esse público, como as doenças sexualmente transmissíveis (DST), principalmente a AIDS; tuberculose; doenças de pele; doenças respiratórias; entre outras. Relata-se, ainda, que o maior problema da área da saúde que atinge os moradores de rua refere-se ao sofrimento mental, como: dependência quími$\mathrm{ca}$, neuroses e psicoses ${ }^{7,29}$. Entre os principais medicamentos utilizados por moradores adultos, masculinos, em situação de rua de Belo Horizonte, encontra-se o psicofármaco, como o principal medicamento usado, seguido de anti-hipertensivo/cardíaco, anti-inflamatório, vitamina, diurético, analgési$\mathrm{CO}$, antibiótico e normoglicemiante ${ }^{28}$. Estudos revelam que os quadros depressivos são companheiros frequentes de quase todas as patologias clínicas crônicas, as quais, quando estão presentes, acabam levando a piores evoluções, pior aderência aos tratamentos propostos, pior qualidade de vida e maior morbimortalidade como um todo. Diversas doenças estão claramente associadas à depressão, com maior destaque para as doenças cardiovasculares, endocrinológicas, neurológicas, renais, oncológicas e outras síndromes dolorosas crônicas ${ }^{30}$. Importante ressaltar que é fundamental o correto tratamento da patologia clínica de base, uma vez que a depressão e as doenças clínicas quase sempre se retroalimentam, interagindo para criar uma situação deteriorante.

Entre as limitações deste estudo, aponta-se que a metodologia utilizada não permite a generalização dos seus achados para outros grupos, pois foi realizado com uma população em situação de rua que procura o centro de referência e não abordou sujeitos em situação de rua que não frequentam esse tipo de serviço. Outro fato é que a depressão foi avaliada exclusivamente com o Inventário de Depressão de Beck. Por fim, preconiza-se o desenvolvimento de outros estudos, tendo em vista tais limites.

\section{CONCLUSÃO}

A condição de morar na rua, especialmente nas grandes e médias cidades, caracteriza hoje um grupo populacional definido no âmbito das políticas sociais como população de rua. Tal grupo vive em condições de extrema pobreza, em geral com precários vínculos familiares e de trabalho e falta de acesso a bens materiais e sociais.

A população de rua deve ser entendida como conjunto de indivíduos sociais, sem trabalho, sem casa, que utiliza a rua como espaço de sobrevivência e moradia. Ser morador de rua não significa apenas estar submetido à condição de espoliação, ao enfretamento de carências, mas significa também adquirir outros referenciais de vida social, diferentes dos anteriores, que eram baseados em valores associados ao trabalho, à moradia e às relações familiares.

Reconhecendo que a depressão também se relaciona com uma série de questões sociais, entre elas fraca inserção no mercado de trabalho, baixa escolaridade, baixa renda, falta de vínculo familiar, condições de moradia precárias, realidade comum da população em situação de rua, podese, então, pensar a prevalência dos quadros depressivos na população masculina adulta em situação de rua de Belo Horizonte como fruto de uma situação de vulnerabilidade social desse grupo social, isto é, a própria condição de vida da população de rua pode vulnerabilizar esse grupo social a maior prevalência de sintomas depressivos.

Se, por um lado, a vulnerabilidade social e econômica pode deixar os indivíduos mais expostos a estados de malestar expressos pela depressão, por outro, o aumento da densidade das redes de apoio social diminui o risco de sofrimento mental. A constatação de que as populações em situação de vulnerabilidade ou de pressão social estão mais sujeitas ao sofrimento mental não faz nem deve fazê-las vítimas de um destino imutável nem de uma realidade estática.

Compreender essa população, suas peculiaridades, sua vida e seus problemas de saúde não resolve o problema da desigualdade e exclusão social. No entanto, acredita-se que a pesquisa pode ser um caminho, como mecanismo de conhecimento e denúncia social, que possibilita a visibilidade dessa situação, a fim de promover ações ou provocar discussões no sentido de estabelecer políticas públicas resolutivas para as iniquidades sociais.

\section{AGRADECIMENTOS}

Os autores agradecem à Fundação de Amparo à Pesquisa do Estado de Minas Gerais (Fapemig) pelo financiamento, processo $n^{\circ} \mathrm{CDS}$ - APQ-00016-08, e pelas bolsas de iniciação científica das acadêmicas: Carolina G. Castro e Ana Karla Silva. Um agradecimento especial ao Centro de Referência da População de Rua (CRPR) de Belo Horizonte pelo apoio na produção científica e a todos os moradores em situação de rua, frequentadores do CRPR, pela participação na pesquisa.

\section{REFERÊNCIAS}

1. Castel R. As metamorfoses da questão social: uma crônica do salário. Petrópolis: Vozes; 1998.

2. Martins JS. Exclusão social e a nova desigualdade. 2. ed. São Paulo: Paulus; 2003.

3. Brasil. Ministério do Desenvolvimento Social e Combate à Fome. Secretaria Nacional de Assistência Social. Política Nacional para Inclusão Social da População em Situação de Rua; 2008. Acesso em: 8 fev. 2009. Disponível em: http://www.coepbrasil.org.br/portal/ Publico/apresentarArquivo.aspx?!D=2954.

4. Silva MLL. Mudanças recentes no mundo do trabalho e o fenômeno população em situação de rua no Brasil 1995-2005 [dissertação]. Braślila: Departamento de Serviço Social/UNB; 2006.

5. Lovisi GM. Avaliação de distúrbios mentais em moradores de albergues públicos das cidades do Rio de Janeiro e de Niterói [tese]. Rio de Janeiro: Escola Nacional de Saúde Pública/Fiocruz; 2000. 
6. Lovisi GM, Lima LA, Morgado AF. População de rua e os transtornos mentais maiores: revisão dos aspectos relevantes. J Bras Psiquiatr. 2001;50(6):149-60.

7. Heckert U, Silva JMF. Psicoses esquizofrênicas entre a população de rua. Rev Psiq Clín. 2002;29(1):14-9.

8. Amado KCO. Avaliação da prevalência de déficits cognitivos e depressão na população idosa residente na Fundação Leão XIII, Rio de Janeiro [dissertação]. Rio de Janeiro: Centro de Ciências Biológicas e da Saúde/UFRJ; 2007.

9. Organização Mundial da Saúde. Relatório sobre a saúde no mundo 2001 - Saúde mental: nova concepção, nova esperança. Genebra: OMS; 2001.

10. Ebert MH, Loosen PT, Nurcombe B. Psiquiatria: diagnóstico e tratamento. Porto Alegre: Artes Médicas; 2002.

11. Organização Mundial da Saúde. Classificação Estatística Internacional de Doenças e Problemas Relacionados à Saúde, 10a revisão. São Paulo: Centro Colaborador da OMS para a Classificação de Doenças em Português; 1995.

12. BRASIL. Ministério do Desenvolvimento Social e Combate à Fome. $2^{\circ}$ Censo da População de Rua e Análise Qual itativa da Situação dessa População em Belo Horizonte. Belo Horizonte: 0 Lutador; 2006.

13. Cunha JA. Manual da versão em português das Escalas Beck. São Paulo: Casa do Psicólogo; 2001.

14. Gorenstein C, Andrade LHSG. Inventário de depressão de Beck: propriedades psicométricas da versão em português. Rev Psiq Clín. 1998;25(5):245-50.

15. Ferreira FPM, Machado S. Vidas privadas em espaços públicos: os moradores de rua em Belo Horizonte. São Paulo: Editora Cortez; 2007.

16. BRASIL. Ministério do Desenvolvimento Social e Combate à Fome. Pesquisa Nacional sobre a População em Situação de Rua Brasil. Braślila: Ministério do Desenvolvimento Social e Combate à Fome; 2008.
17. Rosa CMM. População de rua: Brasil e Canadá. São Paulo: Hucitec; 1995.

18. Holmes DS. Psicologia dos transtornos mentais. Porto Alegre: Artes Médicas; 2001.

19. Seligman M. Estudos experimentais. São Paulo: Hucitec-Edusp; 1977.

20. Gory ML, Ritchey FJ, Mullis J. Depression among the homeless. J Health Soc Behav. 1990; 31(1):87-102.

21. Dooley D, Prause J, Ham-Rowbottom KA. Underemployment and depression: Iongitudinal relationships. J Health Soc Behav. 2000;41(4):421-36.

22. Canônico RP, Tanaka ACD'A, Mazza MMPR, Souza MF, Bernart MC, Junqueira LX. Atendimento à população de rua em um Centro de Saúde Escola na cidade de São Paulo. Rev Esc Enferm USP. 2007;41(n. ${ }^{\circ}$ spe.): 799-803.

23. Boneti LW. Educação, exclusão e cidadania. ljuí: Editora Unijuí; 2003.

24. Escorel S. Vidas ao léu: trajetórias de exclusão social. Rio de Janeiro: Fiocruz; 1999.

25. Bowlby J. Formação e rompimento dos laços afetivos. São Paulo: Martins Fontes; 1997.

26. Dalgalarrondo P. Psicopatologia e semiologia dos transtornos mentais. Porto Alegre: Artes Médicas; 2000

27. Varanda W, Adorno RCF. Descartáveis urbanos: discutindo a complexidade da população de rua e o desafio para políticas de saúde. Saúde Soc. 2004;13(1):56-69.

28. Botti NCL, Castro AG, Ferreira M, Silva AK, Oliveira LC, Castro ACHO, et al. Condições de saúde da população de rua da cidade de Belo Horizonte. Cad Brasil Saúde Mental. 2009;1(2):162-76.

29. Costa APM. População em situação de rua: contextualização e caracterização. Rev Textos Cont. 2005;4(1):1-15.

30. Teng CT, Humes EC, Demetrio FN. Depressão e comorbidades clínicas. Rev Psiq Clín. 2005;32(3):149-59. 